\title{
A Semi-Blind Pilot-Assisted Channel Estimation Algorithm in OFDM Systems
}

\author{
Borching Su \\ Department of Electrical Engineering \\ California Institute of Technology \\ Pasadena, California 91125 \\ Email: borching@systems.caltech.edu
}

\author{
P. P. Vaidyanathan \\ Department of Electrical Engineering \\ California Institute of Technology \\ Pasadena, California 91125 \\ Email: ppvnath@systems.caltech.edu
}

\begin{abstract}
In this paper we study a new semi-blind channel estimation algorithm in orthogonal frequency division multiplexing (OFDM) systems. The proposed scheme is an extension of a recently reported subspace-based blind channel estimation algorithm in cyclic prefix systems which requires very few received blocks. The semi-blind estimation algorithm is devised by using the information obtained both from the blind channel estimation method and a pure pilot-assisted method. The proposed algorithm uses a small amount of received data and can be applied to any types of communication constellations. Simulation results show that, with the same number of pilot samples, the semiblind algorithm has a clear improvement in system performance over the pure pilot-assisted method. To achieve the same biterror-rate performance, the proposed semi-blind algorithm uses fewer pilot samples.
\end{abstract}

\section{INTRODUCTION}

In digital communications, channel estimation is a crucial step for successful data transmission. Channel estimation in block transmission systems has been studied extensively. While pilot-assisted channel estimation uses known pilot samples inserted at the transmitter [5], blind channel estimation which assumes no knowledge of the transmitted samples has also been studied in detail [1]-[4], [9]. Typically, pilot-assisted algorithms are conceptually simple and easy to implement but require some extra bandwidth. In addition, they do not fully exploit the information that is available at the receiver. Blind estimation algorithms, on the other hand, save the bandwidth efficiency but usually are more complicated, require larger amount of received data, and have a poorer system performance than pilot-assisted counterparts. A hybrid of these two types of channel estimation therefore has been studied in an attempt to further improve the system performance.

In this paper, we study semi-blind pilot-assisted channel estimation in orthogonal frequency division multiplexing (OFDM) systems based on cyclic prefixes (CP). Several semiblind algorithms have been proposed for OFDM systems [2], [7]-[10]. However, these methods either rely on the knowledge of source statistics [2], [10] or require the knowledge of source constellation [7]-[9]. Methods relying on source statistics inevitably need to collect a sufficiently large amount of received data to obtain an accurate statistics. This fact makes these methods inapplicable to fast-varying channels since the channel status may have changed significantly by the time

\footnotetext{
${ }^{1}$ Work supported in parts by the NSF grant CCF-0428326 and the Moore Fellowship of the California Institute of Technology.
}

the data is collected. On the other hand, the methods using the knowledge of source constellations usually rely on using a small constellation. When the constellation size is large, these methods become either inapplicable or computationally prohibited. We propose in this paper a semi-blind algorithm based on an extension from a recently reported blind channel estimation algorithm based on subspace decomposition which requires a limited amount of received data. Due to the nature of subspace-based methods [12], the proposed algorithm is applicable with any source constellation and any pilot sample configuration. Furthermore, unlike other subspace-based methods, the proposed algorithm does not require any statistics of input signal and needs much fewer received data than any other subspace-based methods. These features make it suitable for fast-varying channels.

The rest of the paper is organized as follows. In Section II, the system setting is described and the problem statement is formulated. In Section III we first review the previously reported blind and pilot-assied channel estimation algorithms and then present the proposed algorithm. Simulation results are shown in Section IV and the conclusions made in Section $\mathrm{V}$.

\section{A. Notations}

Boldfaced lower case letters represent column vectors. Boldfaced upper case letters and calligraphic upper case letters are reserved for matrices. Superscripts ${ }^{*}, T$, and ${ }^{\dagger}$ as in $a^{*}$, $\mathbf{A}^{T}$, and $\mathbf{A}^{\dagger}$ denote the conjugate, transpose, and transposeconjugate operations, respectively. All the vectors and matrices in this paper are complex-valued. The notation $W_{M}$ denotes $e^{j 2 \pi / M}$, and $\mathbf{W}_{M}$ is the $M \times M$ normalized DFT matrix whose $k l$-th entry is $W_{M}^{-(k-1)(l-1)} / \sqrt{M}$. $\mathbf{I}_{M}$ is the $M \times M$ identity matrix, and $0_{m \times n}$ is the $m \times n$ zero matrix.

Due to the special property of cyclic prefixes, we will use the following notation extensively in this paper. Suppose $\mathbf{y}$ is

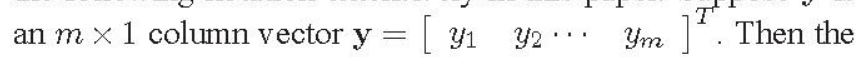
notation $[\mathbf{y}]_{a: b}$ denotes the $(b-a+1) \times 1$ vector

$$
[\mathbf{y}]_{a: b}=\left[\begin{array}{llll}
y_{a} & y_{a+1} & \cdots & y_{b}
\end{array}\right]^{T}
$$

if $1 \leq a \leq b \leq m$. An extension of this definition to any arbitrary pair of integers $a$ and $b$ satisfying $a \leq b$ is made by defining $y_{k}$ as $\left.y_{(k-1} \bmod m\right)+1$ for any $k>m$ or $k<1$. For 
example, if $\mathbf{y}=\left[\begin{array}{lll}y_{1} & y_{2} & y_{3}\end{array}\right]^{T}$, then $[\mathbf{y}]_{-1: 7}$ denotes the vector $\left[\begin{array}{lllllllll}y_{2} & y_{3} & y_{1} & y_{2} & y_{3} & y_{1} & y_{2} & y_{3} & y_{1}\end{array}\right]^{T}$.

\section{Problem Formulation}

Figure 1 shows a cyclic prefix (CP)-based OFDM system. The source data

$$
\mathbf{s}(n)=\left[\begin{array}{llll}
s_{1}(n) & s_{2}(n) & \cdots & s_{M}(n)
\end{array}\right]^{T}
$$

is a sequence of vectors with size $M$ and is transformed into $\mathbf{u}_{M}(n)$ with an inverse FFT operation. The transformed sequence can be expressed as $\mathbf{u}_{M}(n)=\mathbf{W}_{M}^{\dagger} \mathbf{s}(n)$. A cyclic prefix of length $L<M, \mathbf{u}_{c p}(n)$, is defined as the last $L$ elements of $\mathbf{u}_{M}(n)$ and is inserted in the beginning of each block. The signal, after serial-to-parallel operation, is sent over the channel $H(z)$. We assume $H(z)$ is an FIR system with a maximum order $L$, that is,

$$
H(z)=\sum_{k=0}^{L} h_{k} z^{-k} .
$$

Define $\mathbf{h}$ as the $(L+1)$-fold column vector

$$
\left[\begin{array}{llll}
h_{0} & h_{1} & \cdots & h_{L}
\end{array}\right]^{T} .
$$

The received samples $y(n)$ are blocked into vectors $\mathbf{y}(n)$ with length $M+L$. We assume perfect block synchronization between the transmitter and receiver. Denote $\mathbf{y}_{c p}(n)$ as the first $L$ entries and $\mathbf{y}_{M}(n)$ as the last $M$ entries of $\mathbf{y}(n)$. It can be shown that

$$
\mathbf{y}_{M}(n)=\mathbf{H}_{c i r} \mathbf{u}_{M}(n)+\text { noise }
$$

where $\mathbf{H}_{c i r}$ is an $M \times M$ circulant matrix [6] whose first column is $\left[\begin{array}{lllllll}h_{0} & h_{1} & \cdots & h_{L} & 0 & \cdots & 0\end{array}\right]^{T}$. The vector $\mathbf{y}_{M}(n)$ goes through an FFT operation and $\mathbf{x}(n)$ is obtained. Using the property that the DFT matrix $\mathbf{W}_{M}$ diagonalizes circulant matrices, the relationship of vectors $\mathrm{x}(n)$ and $\mathrm{s}(n)$ can be written as

$$
\mathbf{x}(n)=\operatorname{diag}(\mathbf{s}(n))\left[\begin{array}{c}
H\left(e^{j 0}\right) \\
H\left(e^{j 2 \pi / M}\right) \\
\vdots \\
H\left(e^{j 2 \pi(M-1) / M}\right)
\end{array}\right]+\text { noise. }
$$

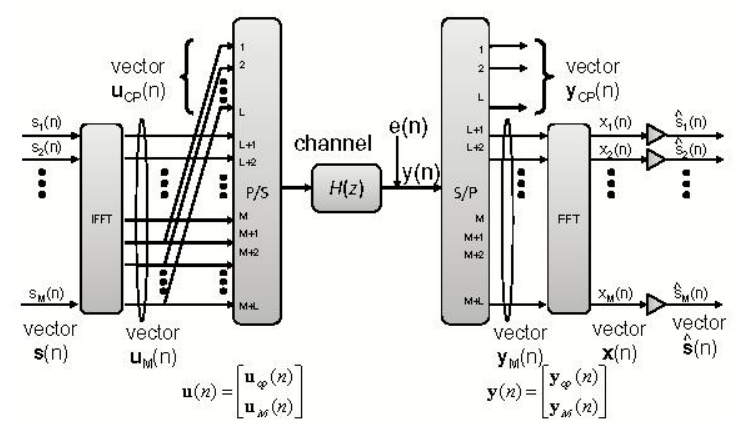

Fig. 1. A CP-based orthogonal frequency division multiplexing (OFDM) system.
Suppose some samples of $\mathbf{s}(n)$ are chosen as pilot samples known to the receiver and do not carry user information. Specifically, define the set of pilot indices

$$
\mathcal{C}_{\text {pil }}=\left\{(m, n) \mid 0 \leq m \leq M-1, n \geq 0, s_{m}(n) \text { is known }\right\} .
$$

Consider the case where each channel estimate is obtained by using $J$ consecutive received blocks $\mathbf{y}(n), n=t, t+1, \ldots, t+$ $J-1$. Let

$$
\mathcal{C}^{(t, J)}=\{(n, m) \mid t \leq n \leq t+J-1,0 \leq m \leq M-1\}
$$

and let $\mathcal{C}_{\text {pil }}^{(t, J)}=\mathcal{C}_{\text {pil }} \cap \mathcal{C}^{(t, J)}$ be the pilot indices in the $J$ blocks. Suppose $\mathcal{C}_{\text {pil }}^{(t, J)}$ has a size $K$ and denote the $k$ th element of $\mathcal{C}_{p z l}^{(t, J)}$ as $\left(n_{k}, m_{k}\right)$. Let

$$
\mathbf{s}_{p i l}=\left[\begin{array}{llll}
s_{m_{1}}\left(n_{1}\right) & s_{m_{2}}\left(n_{2}\right) & \cdots & s_{m_{K}}\left(n_{K}\right)
\end{array}\right]^{T}
$$

be the vector containing all pilot samples in these $J$ blocks. The problem can be formulated as follows. Given $J$ received blocks $\mathbf{y}(n), n=t, t+1, \ldots, t+J-1, \mathbf{s}_{\text {pil }}$, and $\mathcal{C}_{\text {pil }}^{(t, J)}$, how do we estimate the channel coefficients $\mathbf{h}$ ?

\section{Semi-Blind ChanNel Estimation}

The proposed semi-blind algorithm presented in this paper is a combination of a pure pilot-assisted algorithm and a recently reported blind channel estimation algorithm. We will first review both algorithms and then present the proposed algorithms. As shown in Figure 2, all received samples, including the CP parts, will be used for a blind estimation procedure. After FFT operation, received samples at the pilot positions, will be used for pilot-assisted estimation. These results will be combined to obtain an even more accurate estimate than both previously reported algorithms.

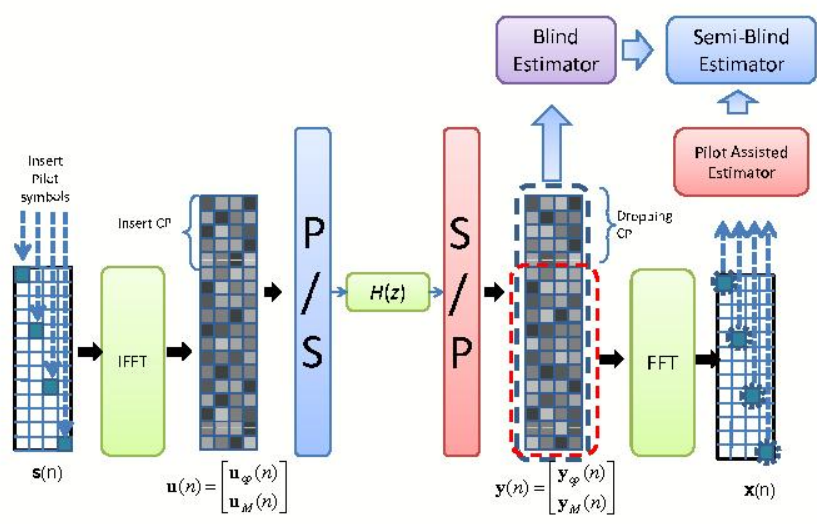

Fig. 2. Illustration of the approach of the proposed semi-blind estimation algorithm.

\section{A. Pure pilot-Assisted Channel Estimation}

A pure pilot-assisted channel estimation method can be deployed (see [5] and the references therein). From (1), it is readily verified that

$$
\mathbf{x}(n)=\sqrt{M} \operatorname{diag}(\mathbf{s}(n)) \mathbf{W}_{M, L+1} \mathbf{h}+\text { noise, }
$$


where $\mathbf{W}_{M L+1}$ is an $M \times(L+1)$ matrix composed of the first $(L+1)$ columns of $W_{M}$. Let $s_{2}$, be the pilot sample vector defined as in (2) and

$$
\mathrm{x}_{m_{i l}}=\left[\begin{array}{llll}
x_{m_{1}}\left(n_{1}\right) & x_{m_{2}}\left(n_{2}\right) & \cdots & x_{m_{K}}\left(n_{K}\right)
\end{array}\right]
$$

be the corresponding received pilot samples. Then from (3), we have

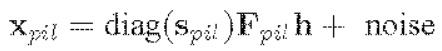

where $F_{p i l}$ is a $K \times(L+1)$ matrix whose $k$ th row is the $m_{\text {sin }}$ th row of $W_{M, L+1}$ and can be expressed as

$$
\left[\begin{array}{llll}
1 & e^{-j 2 \pi r_{k} / M} & \cdots & e^{-j 2 \pi m_{k} L / M}
\end{array}\right] .
$$

A least-square estimate of $h$ is given by

$$
\hat{\mathbf{h}}=\arg \min _{\mathrm{h}}\left\|\mathbf{x}_{p i l}-\operatorname{diag}\left(\mathbf{s}_{p^{i j}}\right) \mathbf{F}_{p_{i j} i} \mathbf{h}\right\|^{2}
$$

\section{B. Blind Channel Estimation}

The pure pilot-assisted algorithm presented above relies on the knowledge of pilot samples at the receiver. In a blind chamel estimation scheme, however, this knowledge is not used except for the purpose of resolving scalar ambiguity. Many blind methods (e.g., [9]) require knowledge of constellations used by the transmitter. These constraints limit the applicability of the methods to constellations with small sizes. When the constellation size is large, these methods become either inapplicable or computationally prohibited. Subspacebased blind methods, on the other hand, do not require any knowledge on source constellation and are applicable with any constellations. Many subspace-based blind methods, however, require a sufficiently large number (usually more than the block size $M$ ) of received blocks to achieve accurate statistics [1] [3]. This constraint makes these subspace-based methods inapplicable in wireless environments where channels are usually fast-varying. A recently reported blind method proposed in [4] generalized the idea of [2] by wisely repeated use of each received blocks, and reduced the amount of actual received data. This property makes this method very promising in a fastvarying chamel environment, at the same time allowing use of any type of constellations, especially a large constellation. We briefly review the algorithm in [4] as follows.

The algorithm reported in [4] has two parameters: 1) the number of available received blocks $J$, and 2) the repetition index $Q$. The parameters must satisfy the inequality

$$
Q(J-2) \geq 2 M-1
$$

While the parameter $J$ represents the actual number of physically received blocks, the repetition index $Q$ can be chosen as any positive integer. As we can see from inequality (6), as long as $Q$ is chosen large enough, the number of received blocks $I$ can be very small. This makes the method work even in time-varying channels. For any two consecutive received blocks $y(n-1)$ and $y(n)$, construct a $(2 M+Q+L-1) \times Q$ matrix

$$
\mathbf{Y}_{Q}(n)=\left[\begin{array}{llll}
\overline{\mathbf{y}}_{0, Q-1}(n) & \overline{\mathbf{y}}_{1, Q-2}(n) & \cdots & \overline{\mathbf{y}}_{Q-1,0}(n)
\end{array}\right]
$$

where

$$
\bar{y}_{k l}(n)=\left[\begin{array}{c}
{\left[\mathrm{y}_{M}(n-1)\right]_{-k+1: M}} \\
\mathrm{y}_{C P}(n) \\
{\left[\mathrm{y}_{M}(n)\right]_{1: M+l}}
\end{array}\right]
$$

for $k, l=0,1, \ldots, Q-1$. It can be shown that [4]

$$
\mathrm{Y}_{Q}(n)=\overline{\mathbf{H}}_{Q} \mathrm{U}_{Q}(n)
$$

where

$$
\overline{\boldsymbol{H}}_{Q}=\left[\begin{array}{ccc}
\boldsymbol{H}_{C r} & 0_{M \times(M+Q \cdots 1)} \\
0_{(L+Q \cdots 1) \times(M-L)} & \mathcal{H}_{L+Q \cdots 1} & 0_{(L+Q \cdots 1) \times(M \cdots L)} \\
0_{M \times(M+Q-1)} & \mathbf{H}_{C 2}
\end{array}\right],
$$

and $\mathrm{U}_{Q}(n)$ is defined as follows.

$$
\mathbf{U}_{Q}(n)=\left[\begin{array}{llll}
\bar{u}_{0, Q-1}(n) & \overline{\mathbf{u}}_{1, Q-2}(n) & \cdots & \overline{\mathbf{u}}_{Q-1,0}(n)
\end{array}\right] .
$$

This is a $(2 M+Q-1) \times Q$ matrix where

$$
\mathbf{u}_{\xi l}(n)=\left[\begin{array}{c}
{\left[\mathbf{u}_{M}(n-1)\right]_{-,+1: M}} \\
{\left[\mathbf{u}_{M}^{\prime}(n)\right]_{1: M+l}}
\end{array}\right]
$$

for $k, l=0,1, \ldots, Q-1$. Suppose $J$ consecutive received blocks $\mathrm{y}(n), n=t, t+1, \ldots, t+J-1$ are available, we can construct a $(2 M+L+Q-1) \times(J-1) Q$ matrix

$\mathbf{Y}_{Q}^{(J)}=\left[\begin{array}{llll}\mathbf{Y}_{Q}(t+1) & \mathbf{Y}_{Q}(t+2) & \ldots & \mathbf{Y}_{Q}(t+J-1)\end{array}\right]$

It is readily verified that

$$
Y_{Q}^{(J)}=\overline{\mathbf{H}}_{Q} \mathrm{U}_{Q}^{(J)}
$$

where

$$
\mathbf{U}_{Q}^{U}=\left[\begin{array}{llll}
\mathbf{U}_{Q}(t+1) & \mathbf{U}_{Q}(t+2) & \cdots & \mathbf{U}_{Q}(t+y-1)
\end{array}\right]
$$

is a $(2 M+Q-1) \times Q(J-1)$ matrix. Note that inequality (6) is a necessary condition (but not sufficient) that $\mathrm{U}_{Q}^{(J)}$ has full rank. However, when $M$ is large, the probability that $U_{Q}^{(J)}$ has full rank is usually very close to unity, especially when a large constellation is used (see [4]). Since $U_{Q}^{(J)}$ has full rank, the range space of $\mathbf{Y}_{Q}^{(J)}$ is equal to that of $\mathbf{H}_{Q}$. So each of $L$ left annihilators of $Y_{Q}^{(3)}$ is also a left annihilator of $\overline{\boldsymbol{H}}_{Q}$. Suppose $\mathrm{g}_{k}, k=1,2, \ldots, L$, is a left amihilator of $\mathbf{Y}_{Q}^{(J)}, 1$.e.,

$$
\mathrm{g}_{k}^{t} \mathbf{Y}_{Q}^{(J)}=\mathrm{g}_{\mathrm{l}}^{\dagger} \mathrm{H}_{Q}=0^{\mathrm{T}}
$$

By exploring each column of $\overline{\mathbf{H}}_{Q}$, a matrix $G_{k}$ can be constructed so that (11) is equivalent to

$$
G_{k} \mathrm{~h}=0 \text {. }
$$

The details of constructing $G_{k}$ from $g_{k}$ is as follows. Let $\mathbf{G}_{k}$ be a Hankel matrix whose first column is $\left[0_{1 \times L}, g_{t 1}^{*}\right]^{T}$ and whose last row is $\left[g_{i}^{\dagger}, 0_{1 \times L}\right]$. Define a sparse matrix

$$
\mathbf{A}=\left[\begin{array}{ccc}
\mathbf{A}_{1}^{T} & \mathbf{I}_{2 N+Q-1} & \mathbf{A}_{2}^{T}
\end{array}\right]^{T}
$$

where $\mathbf{A}_{1}=\left[\begin{array}{lll}0_{L \times(M-L)} & \mathbf{I}_{L} & 0_{L \times(M+Q-1)}\end{array}\right]$ and $\mathbf{A}_{2}=$ $\left[\begin{array}{lll}0_{L \times(M+O-1)} & \mathbf{I}_{L} & 0_{L \times(M-L)}\end{array}\right]$. Then, by simply choosing

$$
G_{k}=\mathbb{A}^{T} \mathrm{G}_{k}^{T}
$$


Eq. (12) is satisfied. Now, by defining

$$
\mathcal{G}=\left[\begin{array}{llll}
\mathcal{G}_{1}^{T} & \mathcal{G}_{2}^{T} & \cdots & \mathcal{G}_{L}^{T}
\end{array}\right]^{T},
$$

we have $\mathcal{G} \mathbf{h}=0$. The channel coefficients $\mathbf{h}$ can be identified within a scalar ambiguity.

\section{Proposed Algorithm}

Note that the blind channel estimation algorithm proposed in Section III-B does not impose any constraints on the input samples $\mathbf{s}(n)$ except for the requirement that $\mathbf{U}_{Q}^{(J)}$ as defined in (10) must have full rank. This property is an advantage in adaption in semi-blind schemes: the positions of pilot samples can be freely chosen and their values do not have to be selected from a given constellation. A semi-blind estimation technique can be devised by using both the information obtained from a blind method and a pilot-assisted method described above. Specifically, we can use the following expression as the objective function for channel estimation:

$$
\left\|\mathbf{x}_{\text {pil }}-\operatorname{diag}\left(\mathbf{s}_{\text {pil }}\right) \mathbf{F}_{\text {pil }} \mathbf{h}\right\|^{2}+\beta\|\mathcal{G} \mathbf{h}\|^{2}
$$

where $\beta \geq 0$ is a constant which can be adjusted according to how much we are relying on the information obtained from blind method and from pilot-assisted method, respectively. The proposed semi-blind algorithm is summarized as follows.

1) Collect $J$ consecutive blocks $\mathbf{y}(n), n=t, t+$ $1, \ldots, t+J-1$, at the receiver and construct a $(2 M+L+Q-1) \times(J-1) Q$ matrix $\mathbf{Y}_{Q}^{(J)}$ as defined in (9).

2) Perform SVD on $\mathbf{Y}_{Q}^{(J)}$ so that

$$
\mathbf{Y}_{Q}^{(J)}=\left[\begin{array}{ll}
\mathbf{U}_{s} & \mathbf{U}_{n}
\end{array}\right]\left[\begin{array}{cc}
\boldsymbol{\Sigma}_{s} & 0 \\
0 & \boldsymbol{\Sigma}_{n}
\end{array}\right]\left[\begin{array}{l}
\mathbf{V}_{s}^{\dagger} \\
\mathbf{V}_{n}^{\dagger}
\end{array}\right]
$$

where the diagonal entries of $\Sigma_{n}$ are the $L$ smallest singular values of $\mathbf{Y}_{Q}^{(J)}$.

3) Let $\mathbf{g}_{k}$ be chosen as the $k$ th column of $\mathbf{U}_{n}$. Construct the $(2 M+Q-1) L \times(L+1)$ matrix $\mathcal{G}$ as in (13).

4) Collect the received pilot samples and form the vector $\mathbf{x}_{\text {pil }}$ as defined in (4). Also construct vector $\mathbf{s}_{\text {pil }}$ and matrix $\mathbf{F}_{\text {pil }}$ as defined in (2) and (5), respectively.

5) Take the estimate $\hat{\mathbf{h}}$ to be the value of $\mathbf{h}$ which minimizes the objective function defined in (14), that is,

$$
\hat{\mathbf{h}}=\arg \min _{\mathbf{h}}\left(\left\|\mathbf{x}_{p i l}-\operatorname{diag}\left(\mathbf{s}_{p i l}\right) \mathbf{F}_{p i l} \mathbf{h}\right\|^{2}+\beta\|\mathcal{G} \mathbf{h}\|^{2}\right) .
$$

\section{Simulation Results}

In this section, we present the simulation results of the proposed semi-blind algorithm. We choose $M=64$ and $L=15$. The data symbols are chosen from a 64-QAM constellation to demonstrate the capability of the proposed algorithm with a large constellation. We use a 16-tap Rayleigh random channel whose power delay profile is defined as in Table I. One thousand realizations of the channel are used in the simulation. We use two different pilot symbol configurations. In configuration 1, as shown in the left part of Figure 3, pilot samples are placed in 16 different frequency bins. In configuration 2, as depicted in the right part of Figure 3, only 12 different frequency bins are used for pilot samples. The pilot samples are chosen so that they are uniformly distributed in an OFDM symbol to achieve the optimal positions [11]. In both configurations the pilot patterns repeat for every 8 OFDM symbols. Note that for a 16-tap channel, a pure pilotassisted scheme requires pilot samples to be placed in at least 16 frequency bins. Each pilot sample has an absolute value $\sqrt{E_{s}}$ where $E_{s}=42$ is the average sample energy for a 64 QAM constellation.

Ten blocks are used for each channel estimate $(J=10)$. Channel estimation is performed for every 5 OFDM symbols, i.e., for the $k$ th channel estimate, $t$ is chosen as $5 k$. The repetition index is chosen as $Q=25$ so that inequality (6) is satisfied. Notice that it is the idea of repetition index that makes it possible to choose the number of blocks as small as 10 . It can be observed that for each channel estimation, $K=20$ pilot samples are available for configuration 1 while $K=15$ for configuration 2 . The parameter $\beta$ defined in (14) is 32 . This value is chosen to give the best system performance based on empirical observations. The performance metric in channel estimation mean square error is defined as

$$
E_{c h}=E\left[\frac{1}{N_{c h}} \sum_{k=0}^{N_{c h}}\left\|\hat{\mathbf{h}}_{k}-\mathbf{h}\right\|^{2}\right],
$$

where the expectation is taken over 1,000 different channel realizations, $N_{c h}$ is the number of total channel estimates for each channel realization, and $\hat{\mathbf{h}}_{k}$ is the $k$ th channel estimate. Figure 4 shows the simulation results. For the least square pure pilot-assisted method, configuration $2(K=15)$ does not work while configuration $1(K=20)$ has a satisfactory performance. The pure blind method works properly but the performance is obviously worse than the pure pilot-assisted method with $K=20$. When a semi-blind technique with $\beta=$ 32 is used, the performances for both $K=20$ and $K=15$ are better than those of pure pilot-assisted method.

Based on the estimated channel coefficients, a linear minimum mean square error (L-MMSE) equalizer is used in OFDM symbol recovery [4]. The $k$ th channel estimate $\hat{\mathbf{h}}_{k}$ is used for equalizing the OFDM symbol numbers $5 k+3$ to $5 k+7$. Equalized symbols then go through detection devices and bit error rate (BER) performance of the system is evaluated which is shown in Figure 5. We see that the semi-blind method with $K=15$ has an even better performance than the pure pilot-assisted method with $K=20$ when the $E_{s} / N_{0}$ ratio is greater than $23 \mathrm{~dB}$. This suggests the proposed semiblind algorithm does reduce the number of pilot samples for achieving the same BER performance.

\section{CONCLUding REMARKS}

In this paper, we proposed a semi-blind channel estimation algorithm in OFDM systems based on a combination of a previously reported blind estimation algorithm in cyclic prefix systems and a pure pilot-assisted algorithm. The proposed algorithm is presumably the first one to be applicable with any types of communication constellations and a limited number of 


\begin{tabular}{c|c|c||c|}
\hline Tap & $\begin{array}{c}\text { Avg. Power } \\
(\mathrm{dB})\end{array}$ & Tap & $\begin{array}{c}\text { Avg. Power } \\
(\mathrm{dB})\end{array}$ \\
\hline 1 & 0.0 & 9 & -6.9 \\
2 & -0.9 & 10 & -7.8 \\
3 & -1.7 & 11 & -4.7 \\
4 & -2.6 & 12 & -7.3 \\
5 & -3.5 & 13 & -9.9 \\
6 & -4.3 & 14 & -12.5 \\
7 & -5.2 & 15 & -13.7 \\
8 & -6.1 & 16 & -18.0 \\
\hline \hline
\end{tabular}

TABLE I

POWER DELAY PROFILE OF THE CHANNEL MODEL USED IN SECTION IV

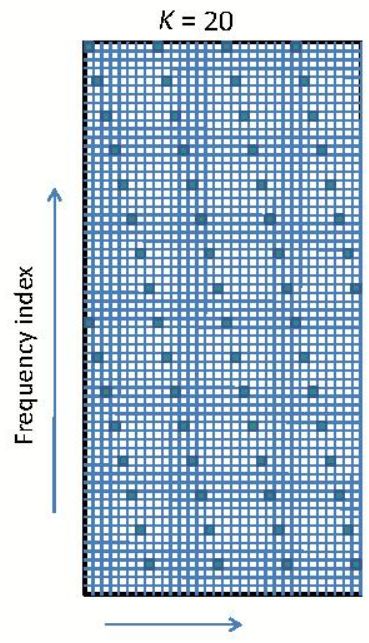

OFDM symbol index

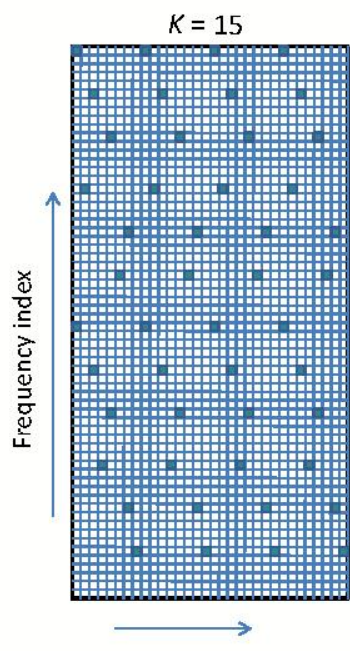

OFDM symbol index
Fig. 3. Pilot positions for $K=20$ (left) and $K=15$ (right).

received blocks. Simulation results confirm the improvement in system performance of the semi-blind algorithm over the direct pilot-assisted algorithm. They also suggest that fewer pilot samples can be used to achieve the same BER performance when a semi-blind algorithm is employed.

In the future, many aspects are worthy of further investigation. For example, it may be interesting to analytically derive the optimal parameter $\beta$. The optimal design of the pilot symbol configurations (i.e., pilot position, value, etc.) for the semi-blind algorithm is still an unknown but important issue.

\section{REFERENCES}

[1] X. Cai and A. Akansu, "A subspace method for blind channel identification in OFDM systems," in Proc. Int. Conf. Commun., vol 2, New Orleans, LA, pp. 929-933, Jun. 2000.

[2] B. Muquet, M. de Courville, and P. Duhamel, "Subspace-based blind and semi-blind channel estimation for OFDM systems," IEEE Trans. Signal Process., vol. 50, no. 7, pp. 1699-1712, Jul. 2002.

[3] C. Li and S. Roy, "Subspace-based blind channel estimation for OFDM by exploiting virtual carriers," IEEE Transactions on Wireless Communications, vol. 2 , no. 1 , pp. 141-150, Jan. 2003.

[4] B. Su and P. P. Vaidy anathan, "Subspace-based blind channel identification for cyclic prefix systems using few received blocks," IEEE Trans. Signal Process. vol. 55, no. 10, pp. 4979-4993, Oct. 2007.

[5] L. Tong, B. M. Sadler, and M. Dong, "Pilot-assisted wireless transmissions: general model, desion criteria, and signal processing," IEEE Signal Processing Magazine, vol. 21, no. 6, pp. 12-15, Nov. 2004.

[6] P. P. Vaidyanathan, Multirate Systems and Filter Banks, Prentice-Hall, 1993.

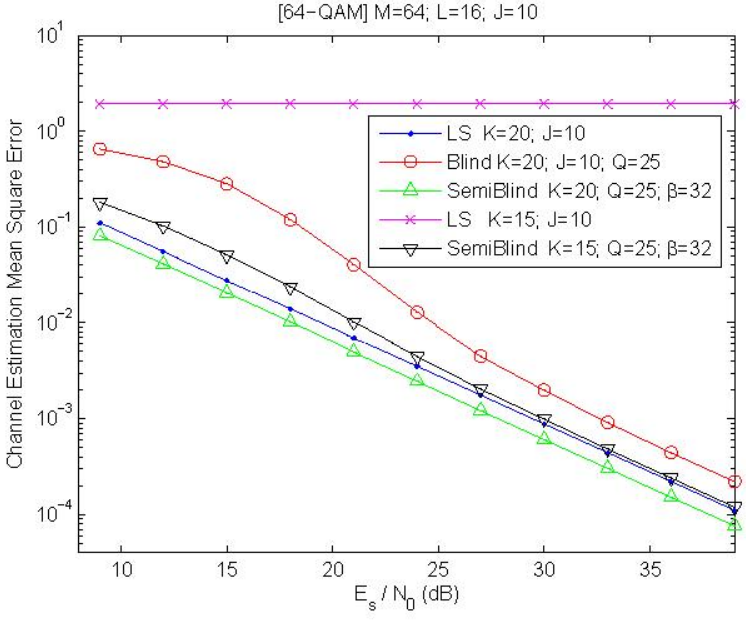

Fig. 4. Comparison of pilot-based and semi-blind methods in channel estimation mean square error performance.

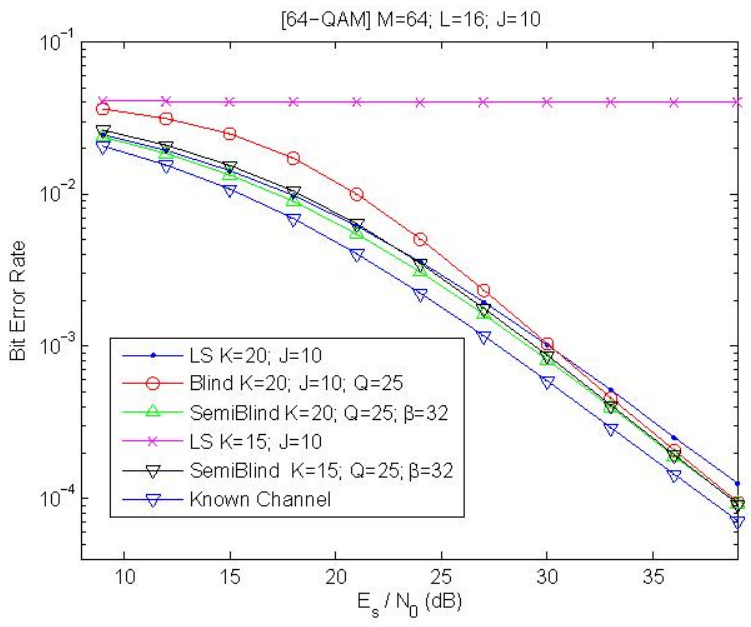

Fig. 5. Comparison of pilot-based and semi-blind methods in bit error rate performance.

[7] K. Y. Ho and S. H. Leung, "A generalized semi-blind channel estimation for pilot-aided OFDM systems," in Proc. ISCAS 2005, Kobe, Japan, May 2005.

[8] W. Yang, Y. Cai, and Y. Xun, "Semi-blind channel estimation for OFDM systems," in Proc. IEEE Vehicular Technology Conference, 2006. VTC 2006-Spring.

[9] S. Zhou and G. Giannakis, "Finite-alphabet based channel estimation for OFDM and related multicarrier systems," IEEE Transactions on Wireless Communications, vol. 49, no. 8, pp. 1402-1414, Aug. 2001.

[10] T. Cui and C. Tellambura, "Semi-blind channel estimation and data detection for OFDM systems," in Proc. ICASSP 2005, Philadelphia, PA.

[11] S. Adireddy, L. Tong, and H. Viswanathan, "Optimal placement of training for frequency-selective block-fading channels," IEEE Trans. on Information Thoery vol. 48, no. 8, Aug. 2002.

[12] E. Moulines, P. Duhamel, J. F. Cardoso, S. Mayrargue, "Subspace Methods for the Blind Identification of Multichannel FIR Filters," IEEE Trans. on SP, pp. 516-525, Feb. 1995. 